\title{
Study of Course Design for Electromechanical System in College Education
}

\author{
Yi Jiangang ${ }^{1,2, a^{*}}$ \\ ${ }^{1}$ Jianghan University, School of Electromechanical and Architectural Engineering, Wuhan, 430056, \\ China \\ 2 Jianghan University, Wuhan Engineering Research Center of Complex Electromechanical \\ System, Wuhan, 430056, China \\ ajg_wh@yeah.net
}

\begin{abstract}
Keywords: Electromechanical system, Course design, Large grinder, System upgrades
Abstract. Course design for electromechanical system is one of the most important and difficult points in college teaching process. In this paper, the large grinder recirculation cooling system as the sample, the hydraulic cooling system is built to solve the problems of low cooling efficiency, high failure rate and oil pollution in the old system. Based on it, the combination of PLC control and manual control is discussed, a special pipe heater and the oil temperature monitoring system are designed, and the design process of the course design for electromechanical system is introduced, which could be used as a good reference to guide students for course design.
\end{abstract}

\section{Introduction}

Course design for electromechanical system is one of the most important and difficult points in college teaching process. During design process, students wonder how to find appropriate design object because of their lacking experience. Furthermore, once the design titles are present by teachers, students find it difficult to locate the existing problems of the giving objects. Therefore, the large grinder recirculation cooling system as the sample, we discuss the method of Course design for electromechanical system in this paper.

\section{Existing Problems}

According to the practical investigation of production situation, we find there exist the following problems when using the large grinder:

(1) Due to the machining characteristics and the fuel tank position, some debris will inevitably be mixed with oil during the processing of working. On the other hand, there lacks of precision filtering system in the original lubricating system, which caused the problems such as low oil cleanliness, nozzle clogging, wear of pump body with oil pan, damage of the sealing ring. These problems often lead to faults of the old recirculation cooling system.

(2) Because of the viscosity temperature characteristics of hydraulic oil, the lower the temperature, the higher the viscosity of the oil. Therefore, under the condition of low temperature, it is difficult for hydraulic pump to absorb oil, which results in the failure of forming film lubrication between guide and working table because the lubricating oil cannot be spread successfully. This may cause the reduction of the machining precision and the wear of the grinder.

(3) The temperature of hydraulic oil plays a vital role for the performance and machining precision of the grinder. However, the large grinder had not set the oil temperature control system. Much of the cutting heat produced by grinding generated by the device itself will be sent to the hydraulic oil, which will result in the high temperature of the oil under a long time, thereby reducing the service life and the quality of the oil. 


\section{Design Solutions}

In view of the above problems exposed in the actual production of the large grinding machine, we focus on the improvement of oil cleanliness and temperature control of the old system. Combined with the design features of hydraulic system, it is decided to set up a separate set of oil pump motor to form a new hydraulic circulating cooling system. The principle diagram of the hydraulic circulating cooling system is shown in Figure 1. The old oil source of the large grinder is still used. The new hydraulic system is composed of gear pump 1, filter 2 , heater 3 , reversing valve 4 , cooler 5 , oil tank 6 and other accessories. The pump 1 is used to supply oil and provide the circulation cooling fluid and the filter medium for the system. In order to meet the needs of the actual working conditions, the circulating cooling system is used in the combination of wind cooling and water cooling. When the temperature is high, the valve and the electromagnetic water valve 4 are opened, and the wind cooler is worked. When the temperature is low, the valve 4 can be closed, and the oil can flow back to the oil tank 6 directly from the bypass. In order to ensure the accuracy of the oil filter, the precision filter is set up on the way back to the oil path 2 .

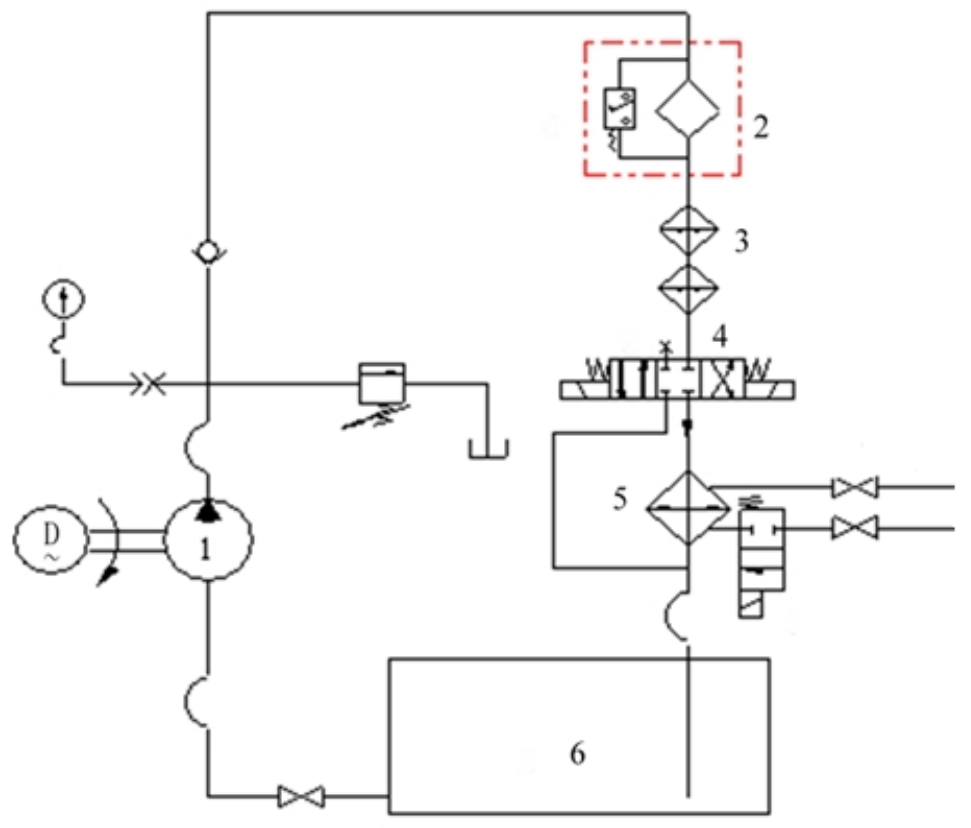

1. Gear pump; 2. Filter; 3. Heater; 4. Reversing valve; 5. Cooler; 6. Oil tank

Figure 1. Hydraulic circulating cooling system

\section{Oil Temperature Monitoring System}

Oil cooling control is very important to the cooling effect of the hydraulic circulating system. In order to accurately control the oil temperature, a set of temperature monitoring system is set up. The oil tank of the original system is used for economy. The heater 3 is set on the circulating cooling system to ensure oil is not frozen in low temperature, and to reduce the influence of the original system. Because of the space restriction of the site, it is inconvenient to install the heater in the old oil box. In order to solve this problem, we have developed a special pipe heater, whose structure is shown in Figure 2. The oil goes through the $U$ shaped pipe from the oil inlet to be heated, and flows out from the oil outlet. In order to ensure the precise control of the oil temperature, four point temperature monitoring method is used, which means four temperature points in four different locations of the oil tank are set through temperature sensors for real-time monitoring. Then, the monitoring data are sent through wires connected to the corresponding display instruments. In order to ensure automatic control of cooling and heating, PLC is used to connect the reversing valve, the cooler, the heater and the corresponding joints. When the temperature is higher than the set temperature, the cooler is worked. When the oil temperature is reduced to the set temperature, the heater worked. The control system block diagram is shown in Figure 3. 


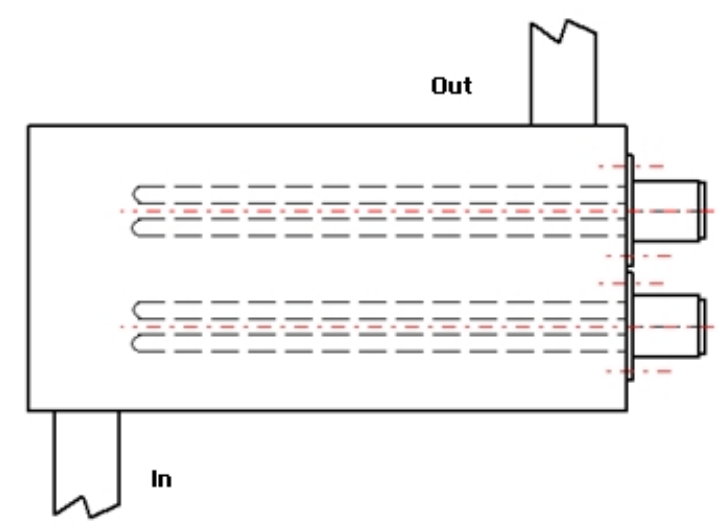

Figure 2. The structure of the heater

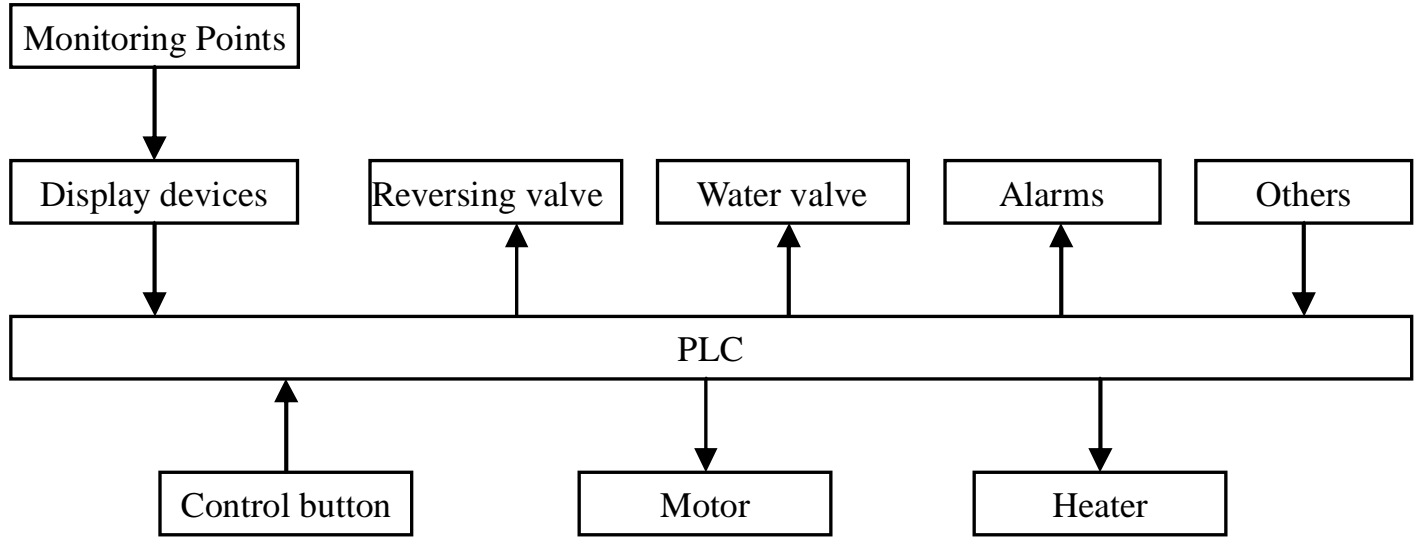

Figure 3. Oil temperature monitoring system

The monitoring operation platform mainly consists of the following parts: digital display devices, system running status indicators, operation buttons and alarms. In the digital display devices module, there are temperature monitoring data and the filter data. The system running state indicators are used to display the important parameters of the pressure and the flow parameters. In order to improve the reliability of the hydraulic circulating cooling system, mechanical and electrical control parts are used on the oil temperature monitor system at the same time. All the monitoring parameters can be displayed on the instruments of the control platform. Heating, cooling, starting and stopping the system can be operated by the control buttons on the platform. At the same time, according to the work site requirements, programs can be developed and downloaded into the PLC to control the motor start and stop, oil heating and cooling, filter blocking alarm, etc., so as to achieve the purpose of daily unattended.

\section{Conclusions}

In this course design sample, the aim is to solve the problem that the original grinder hydraulic cooling system has low efficiency, high failure rate. A new hydraulic circulating cooling system is designed by using the combination of automatic monitoring and manual monitoring methods, and the cooling circulatory system of hydraulic oil temperature monitoring device based on PLC is developed. The idea and the study process of course design of electromechanical system are explained, which have a good reference value to guide students for design work.

\section{Acknowledgements}

This work is supported by Wuhan Engineering Research Center of Complex Electromechanical System and Industry-university-research project of Wuhan Education Bureau (Granted No: cxy02), 
China. The author also gratefully acknowledges the helpful comments and suggestions of the reviewers, which have improved the presentation.

\section{References}

[1] Ho, T.H. and K.K. Ahn, Modeling and simulation of hydrostatic transmission system with energy regeneration using hydraulic accumulator. Journal of Mechanical Science and Technology, 24 (2010) 1163-1175.

[2] Kim, H.E., G.C. Lee and J.H. Kim, System design and performance test of hydraulic intensifier. Transactions of the Korean Society of Mechanical Engineers, 34 (2010), 947-952.

[3] Truong Dinh Quang, Ahn Kyoung Kwan, Force control for hydraulic load simulator using self-tuning grey predictor-fuzzy PID, Mechatronics, 19 (2009), 233-246.

[4] Yi Jiangang. Modelling and Analysis of Step Response Test for Hydraulic Automatic Gauge Control. Strojniški vestnik - Journal of Mechanical Engineering. 61(2015) 115-122

[5] Cheng Nan, Zhu Yan-Zhi. Optimal sequencing and scheduling of middle-sized hydroelectric stations based on fuzzy integral weighting integer programming. System Engineering Theory and Practice, 28 (2008), 122-128. 